\title{
Shedding is no easy task
}

It is not clear whether tumour cells are able to disseminate and begin the process of metastasis early or late during tumour progression. There is unlikely to be one answer that fits all, but understanding the metastatic process is important when considering patient treatment.

Caswell, Chuang et al. sought to ascertain whether early-stage or late-stage lung carcinomas are able to disseminate using $\mathrm{Kras}^{\text {LSL-G12D/+ }}$; $\operatorname{Trp} 53^{\text {flox } x \text { flox }} ;$ Rosa2 $6^{\text {LSL-tdTomato }}$ (KPT) genetically engineered mice that, after inhalation of viral vectors that express Cre recombinase, develop red fluorescently labelled lung tumours in a stepwise manner. 'KPT-late' mice received low amounts of viral Cre and developed 10-50 tumours with evidence of invasion of the surrounding tissue, and 'KPT-early' mice received higher amounts of Cre to induce a higher number of early stage

There is unlikely to be one answer that fits all hyperplastic lesions. Having established that the number of neoplastic cells in both sets of mice were roughly the same, the authors compared them. KPT-early mice had few disseminated tumour cells (DTCs) in the pleural cavity (an early site of lung cancer dissemination),

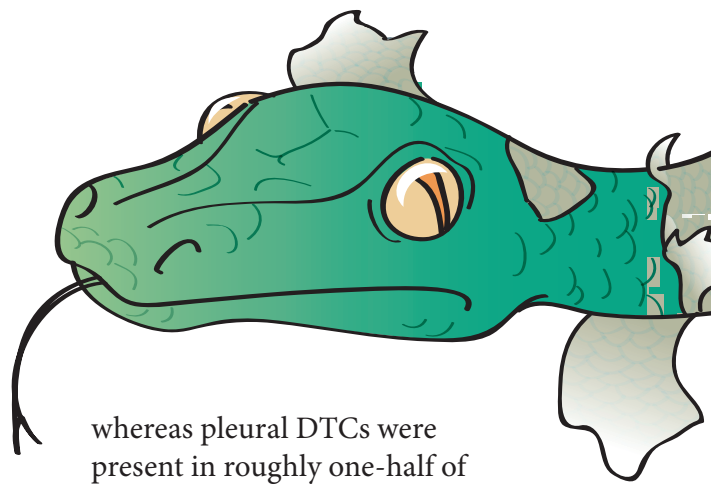

present in roughly one-half of the KPT-late mice. When DTCs were present, the number of DTCs correlated with the number of metastases but did not correlate with the total tumour burden. Further analysis revealed that DTCs in the pleural cavity were derived from a single primary tumour, indicating that the ability to disseminate is a rarely acquired property.

To assess whether the loss of p53 affects dissemination, the authors generated KT mice (which are p53 proficient). KT-late mice developed large primary tumours and only one of $14 \mathrm{KT}$-late mice had pleural DTCs. This indicates that p53 loss is important for dissemination and metastasis of lung cancer cells, but as almost onehalf of the KPT-late mice did not have pleural DTCs, p53 loss is not sufficient for dissemination in this model. expression of this transcription factor in matched primary tumours, DTCs and metastases from KPT-late mice. Nkx2-1 was downregulated in DTCs compared with the primary tumours in eight of nine matched samples, which suggests that this alteration precedes dissemination.

This paper shows that lung carcinomas probably do not have an inherent ability to disseminate. Understanding more clearly what events lead to tumour cell dissemination may shed light on therapeutic approaches to prevent metastasis.

\section{Gemma K. Alderton}

ORIGINAL RESEARCH PAPER Caswell, D. R. et al. Obligate progression precedes lung adenocarcinoma dissemination. Cancer Discov. http://dx.doi.org/10.1158/2159-8290.CD-13-0862 (2014) 